\begin{tabular}{|c|l|}
\hline Title & On implicit second order PDE of a scal ar function on a plane via differential systems \\
\hline Author(s) & Shibuya, Kazuhiro; Noda, Takahiro \\
\hline Citation & Hokkaido University Preprint Series in Mathematics, 939, 1-19 \\
\hline Issue Date & 2009-3-5 \\
\hline DOI & 10.14943/84087 \\
\hline Doc URL & http://hdl.handle.net/2115/69747 \\
\hline Type & bulletin (article) \\
\hline File Information & pre939.pdf \\
\hline
\end{tabular}

Instructions for use 


\title{
ON IMPLICIT SECOND ORDER PDE OF A SCALAR FUNCTION ON A PLANE VIA DIFFERENTIAL SYSTEMS
}

\author{
TAKAHIRO NODA AND KAZUHIRO SHIBUYA
}

\section{INTRODUCTION}

In this paper, we will study implicit second order PDEs for one unknown function of two variables using differential systems.

First of all, we prepare some terminology and notations. Let $J^{2}\left(\mathbb{R}^{2}, \mathbb{R}\right)$ be the 2-jet space:

$$
J^{2}\left(\mathbb{R}^{2}, \mathbb{R}\right):=\{(x, y, z, p, q, r, s, t)\} .
$$

This space has the canonical differential system (or higher order contact system) $C^{2}=$ $\left\{\varpi_{0}=\varpi_{1}=\varpi_{2}=0\right\}$ given by the following 1-forms:

$$
\begin{aligned}
& \varpi_{0}:=d z-p d x-q d y, \\
& \varpi_{1}:=d p-r d x-s d y, \\
& \varpi_{2}:=d q-s d x-t d y .
\end{aligned}
$$

That is, the system $C^{2}$ is a subbundle of the tangent bundle $T J^{2}\left(\mathbb{R}^{2}, \mathbb{R}\right)$. On the 2-jet space, we consider PDEs of the form:

$$
F(x, y, z, p, q, r, s, t)=0
$$

where, the function $F$ is a $C^{\infty}$ function on $J^{2}\left(\mathbb{R}^{2}, \mathbb{R}\right)$. We put

$$
\Sigma=\{F=0\} \subset J^{2}\left(\mathbb{R}^{2}, \mathbb{R}\right)
$$

and restrict the canonical differential system $C^{2}$ to $\Sigma$. We denote $D:=\left.C^{2}\right|_{\Sigma}$. In this situation, there are some cases as follows:

(I) $\Sigma$ is regular (i.e. $\left(F_{r}, F_{s}, F_{t}\right)_{w} \neq(0,0,0)$.) and $\Sigma$ is locally hyperbolic, elliptic or parabolic. (See, section 2)

(II) $\Sigma$ is regular (i.e. $\left(F_{r}, F_{s}, F_{t}\right)_{w} \neq(0,0,0)$.) and $\Sigma$ is not locally parabolic.

(III) $\Sigma$ is a smooth hypersurface. The restriction $D$ is a differential system, but $\Sigma$ has a nonsubmersion point $w \in \Sigma$. Namely, $C^{2}$ is transverse to $T \Sigma$ in $T J$.

(IV) $\Sigma$ is a smooth hypersurface, but $D$ is not a differential system. (This condition is equivalent to $C_{w}^{2} \subset T_{w} \Sigma$.) That is, $D$ is not a subbundle of $T \Sigma$. Moreover, the case (IV)

2000 Mathematics Subject Classification. 2000MSC Primary 58A15; Secondary 57S20.

Key words and phrases. implicit second order PDE, differential system, contact invariant.

First author supported by Research Fellowships of the Japan Society for the Promotion of Science for Young Scientists. 
is divided into the following two cases.

$(\mathrm{IV}-1) D$ is not a differential system and $\left.C^{1}\right|_{\Sigma}$ is a differential system, where $C^{1}:=\partial C^{2}=$ $C^{2}+\left[C^{2}, C^{2}\right]$.

$(\mathrm{IV}-2) D$ is not a differential system and $\left.C^{1}\right|_{\Sigma}$ is not a differential system.

(V) $\Sigma$ is an algebraic variety. (i.e. $\Sigma$ has singular points.)

In works by many researchers (Lie, Cartan, Darboux Monge etc,), almost results are obtained under the regularity condition (I). ([Car]) Then, $\Sigma$ is a (smooth) hypersurface. Also, the restriction $\left.\pi\right|_{\Sigma}: \Sigma \rightarrow J^{1}\left(\mathbb{R}^{2}, \mathbb{R}\right)$ of the natural projection $\pi: J^{2}\left(\mathbb{R}^{2}, \mathbb{R}\right) \rightarrow$ $J^{1}\left(\mathbb{R}^{2}, \mathbb{R}\right)$ is a submersion. (i.e. differential maps are surjective.) By the property, restricted 1-forms $\left.\varpi_{i}\right|_{\Sigma}$ on $\Sigma$ are linear independent. Hence, we have the induced differential system $D=\left\{\left.\varpi_{0}\right|_{\Sigma}=\left.\varpi_{1}\right|_{\Sigma}=\left.\varpi_{2}\right|_{\Sigma}=0\right\}$ on $\Sigma$. The system $D$ is a vector bundle of rank 4 on $\Sigma$.

REMARK 1.1. From now on, we omit the notation $\left.\right|_{\Sigma}$ from generator 1-forms of $D$.

For second order PDEs satisfying the regularity condition, many ample results are obtained via Cartan's methods. Also, Tanaka theory is very strong tool under the regularity condition.([Tan1], [Tan2])

Recently, a topic related to the case (II) is treated in ([CKW], [NS]).

In the present note, we consider the case (III). Precisely speaking, we assume the condition:

Assumption 1.2. Let $\Sigma=\{F=0\}$ be a second order PDE. There exist a nonsubmersion point $w \in \Sigma$ for $\left.\pi\right|_{\Sigma}$ which satisfies the following condition. $\Sigma$ is a smooth hypersurface, and $D:=C^{2}$ is a differential system around $w$. (In general, $\left.\pi\right|_{\Sigma}$ is not local nonsubmersion. See Remark 3.12.)

For ODEs of type (III), there are works. ([HIIY], [Tak]) In case (III), we have some PDEs of special type as examples. The our purpose is to characterize differential systems (or PDEs) satisfying the assumption. Under the assumption, we can define the graded Lie algebra at each point which called symbol algebra in the sense of N. Tanaka. The symbol algebra is a fundamental invariant of differential systems. Our first main result is to characterize symbol algebra at nonsubmersion point:

Theorem 3.10 Let $\Sigma=\{F=0\}$ be a smooth hypersurface of $J^{2}\left(\mathbb{R}^{2}, \mathbb{R}\right)$. Assume that $\Sigma$ has a nonsubmersion point $w \in \Sigma$ for $\left.\pi\right|_{\Sigma}$, and $D:=\left.C^{2}\right|_{\Sigma}$ is a differential system around w. Then, the symbol algebra $\mathfrak{m}(w)$ at $w$ is locally isomorphic to $\mathfrak{m}, \mathfrak{m}=\mathfrak{g}_{-3} \oplus \mathfrak{g}_{-2} \oplus \mathfrak{g}_{-1}$ and bracket relations are given by;

$\left[X_{r}, X_{x}\right]=X_{1}, \quad\left[X_{s}, X_{x}\right]=X_{2}, \quad\left[X_{1}, X_{x}\right]=X_{0}$

the other is trivial, 
where, $\left\{X_{0}, X_{1}, X_{2}, X_{x}, X_{r}, X_{s}, X_{t}\right\}$ are basis, and

$$
\begin{aligned}
& \mathfrak{g}_{-1}=\left\{X_{x}, X_{r}, X_{s}, X_{t}\right\}, \\
& \mathfrak{g}_{-2}=\left\{X_{1}, X_{2}\right\}, \\
& \mathfrak{g}_{-3}=\left\{X_{0}\right\} .
\end{aligned}
$$

According to the result, we can not discriminate differential systems using symbol algebras. From the fact, we need to other tools to research differential system in our case. We will define a new invariant of differential systems associated with second order PDEs. Using the invariant, we study some equations as examples. Consequently, we will determine the values of invariants for PDEs of the special form as second main result. (Theorem 4.7)

Acknowledgment. The authors would like to thank Professor Keizo Yamaguchi for helpful discussions and encouragement.

\section{Review of the REgular PDE}

In this section, we recall basic results for regular PDEs which called the type (I), (II) PDEs as in the introduction. (See, [BCG3], [Y3], [Y4].) Let $\Sigma=\{F=0\} \subset J^{2}\left(\mathbb{R}^{2}, \mathbb{R}\right)$ be a regular $\mathrm{PDE}$, that is $\left(F_{r}, F_{s}, F_{t}\right) \neq(0,0,0)$. Then, we have the following fundamental properties.

(i) $\left.\pi\right|_{\Sigma}: \Sigma \rightarrow J^{1}\left(\mathbb{R}^{2}, \mathbb{R}\right)$ is a submersion.

(ii) $D=\left.C^{2}\right|_{\Sigma}$ is a differential system of rank 4 on $\Sigma$.

(iii)

$$
\begin{aligned}
& D \subset \partial D \subset \partial^{2} D=T \Sigma, \\
& D \subset \partial D \subset \partial^{(2)} D=T \Sigma .
\end{aligned}
$$

where, rank $\partial D=6$. Here, we define the derived system and weak derived system of a differential system $D$ in general. The derived system $\partial D$ is defined, in terms of sections, by

$$
\partial \mathcal{D}:=\mathcal{D}+[\mathcal{D}, \mathcal{D}]
$$

where, $\partial \mathcal{D}=\Gamma(D)$ denotes the space of sections of $D$. In general $\partial D$ is obtained as a subsheaf of the tangent sheaf of $\Sigma$. Moreover higher derived systems $\partial^{k} D$ are defined successively by

$$
\partial^{k} \mathcal{D}:=\partial\left(\partial^{k-1} D\right)
$$

where we put $\partial^{0} D=D$ by convention. On the other hand, weak derived systems $\partial^{(k)} D$ of $D$ are defined successively by

$$
\partial^{(k)} \mathcal{D}:=\partial^{(k-1)} \mathcal{D}+\left[\mathcal{D}, \partial^{(k-1)} \mathcal{D}\right] .
$$

Definition 2.1. A differential system $D$ is called regular (weakly regular), if $\partial^{k} D$ (resp. $\partial^{(k)} D$ ) is subbundle for all $k$. 
REMARK 2.2. Let $D=\left\{\varpi_{1}=\cdots=\varpi_{s}=0\right\}$ be a differential system on a manifold $R$. We denote by $D^{\perp}$ the annihilator subbundle of $D$ in $T^{*} R$, namely,

$$
\begin{aligned}
D^{\perp}(x) & =\left\{\omega \in T_{x}^{*} R \mid \omega(X)=0 \text { for any } X \in D(x)\right\} \\
& =<\varpi_{1}, \cdots, \varpi_{s}>.
\end{aligned}
$$

Then the annihilator $(\partial D)^{\perp}$ of the first derived system of $D$ is given by

$$
(\partial D)^{\perp}=\left\{\varpi \in D^{\perp} \mid d \varpi \equiv 0\left(\bmod D^{\perp}\right)\right\} .
$$

Moreover the annihilator $\left(\partial^{(k+1)} D\right)^{\perp}$ of the $(k+1)$-th weak derived system of $D$ is given by

$$
\begin{aligned}
\left(\partial^{(k+1)} D\right)^{\perp}=\left\{\varpi \in\left(\partial^{(k)} D\right)^{\perp} \mid\right. & d \varpi \equiv 0\left(\bmod \left(\partial^{(k)} D\right)^{\perp},\right. \\
& \left.\left.\left(\partial^{(p)} D\right)^{\perp} \wedge\left(\partial^{(q)} D\right)^{\perp}, 2 \leq p, q \leq k-1\right)\right\} .
\end{aligned}
$$

(iv) The Cauchy characteristic system $C h(D)$ of $D=\left.C^{2}\right|_{\Sigma}$ is trivial bundle.

(v) The Cauchy characteristic system of the first derived system of $D$ is $\operatorname{ker}\left(\left.\pi\right|_{\Sigma}\right)_{*}$. (i.e. rank $C h(\partial D)=2$.) Here, the Cauchy characteristic system $C h(D)$ of a differential system $D$ on $\Sigma$ is defined by

$$
\left.C h(D)(x):=\{X \in D(x) \mid X\rfloor d \varpi_{i} \equiv 0 \quad\left(\bmod \varpi_{0}, \varpi_{1}, \varpi_{2}\right) \quad \text { for } i=0,1,2\right\},
$$

where, $\rfloor$ denotes the interior product (i.e., $X\rfloor d \varpi(Y)=d \varpi(X, Y)$.), and $D=\left\{\varpi_{0}=\varpi_{1}=\varpi_{2}=0\right\}$ is defined locally by defining 1 -forms $\left\{\varpi_{0}, \varpi_{1}, \varpi_{2}\right\}$. When $C h(D)$ is a differential system (i.e. has constant rank), it is always completely integrable.

It is well-known that the regular PDEs are divided into three cases. (hyperbolic, elliptic, parabolic.) For regular second order PDEs $\Sigma:=\{F=0\}, \Sigma$ is said to be hyperbolic, elliptic, or parabolic according to whether the discriminant $\Delta:=F_{s}^{2}-\frac{1}{4} F_{r} F_{t}$ is positive, negative, or zero respectively.

If $\Sigma$ is hyperbolic at $w$, then there exists a local coframe $\left\{\theta_{0}, \theta_{1}, \theta_{2}, \eta_{1}, \eta_{2}, \pi_{1}, \pi_{2}\right\}$ around $w$ such that followings hold at $w$.

$$
\begin{aligned}
D=\left\{\theta_{0}=\theta_{1}\right. & \left.=\theta_{2}=0\right\}, \partial D=\left\{\theta_{0}=0\right\}, \partial^{2} D=T \Sigma \\
d \theta_{0} & \equiv \eta_{1} \wedge \theta_{1}+\eta_{2} \wedge \theta_{2} \quad \bmod \theta_{0} \\
d \theta_{1} & \equiv \eta_{1} \wedge \pi_{1} \quad \bmod \theta_{0}, \theta_{1}, \theta_{2} \\
d \theta_{2} & \equiv \eta_{2} \wedge \pi_{2} \quad \bmod \theta_{0}, \theta_{1}, \theta_{2}
\end{aligned}
$$

If $\Sigma$ is elliptic, then there exists a local coframe $\left\{\theta_{0}, \theta_{1}, \theta_{2}, \eta_{1}, \eta_{2}, \pi_{1}, \pi_{2}\right\}$ around $w$ such that followings hold at $w$.

$$
D=\left\{\theta_{0}=\theta_{1}=\theta_{2}=0\right\}, \partial D=\left\{\theta_{0}=0\right\}, \partial^{2} D=T \Sigma .
$$




$$
\begin{array}{ll}
d \theta_{0} \equiv \eta_{1} \wedge \theta_{1}+\eta_{2} \wedge \theta_{2} & \bmod \theta_{0}, \\
d \theta_{1} \equiv \eta_{1} \wedge \pi_{1}+\eta_{2} \wedge \pi_{2} & \bmod \theta_{0}, \theta_{1}, \theta_{2} \\
d \theta_{2} \equiv \eta_{1} \wedge \pi_{2}+\eta_{2} \wedge \pi_{1} & \bmod \theta_{0}, \theta_{1}, \theta_{2}
\end{array}
$$

If $\Sigma$ is parabolic, then there exists a local coframe $\left\{\theta_{0}, \theta_{1}, \theta_{2}, \eta_{1}, \eta_{2}, \pi_{1}, \pi_{2}\right\}$ around $w$ such that followings hold at $w$.

$$
\begin{aligned}
& D=\left\{\theta_{0}=\theta_{1}=\theta_{2}=0\right\}, \partial D=\left\{\theta_{0}=0\right\}, \partial^{2} D=T \Sigma \\
& d \theta_{0} \equiv \eta_{1} \wedge \theta_{1}+\eta_{2} \wedge \theta_{2} \quad \bmod \theta_{0}, \\
& d \theta_{1} \equiv \eta_{1} \wedge \pi_{1} \quad \bmod \theta_{0}, \theta_{1}, \theta_{2} \\
& d \theta_{2} \equiv \eta_{1} \wedge \pi_{2}+\eta_{2} \wedge \pi_{1} \quad \bmod \theta_{0}, \theta_{1}, \theta_{2}
\end{aligned}
$$

REMARK 2.3. The above discussions are pointwise. But if, $\Sigma$ is hyperbolic or elliptic, then these results can be extended to local structure. There is a possibility that parabolic case has a certain singularities as in the next example.

ExAmPLE 2.4. This example shows the existence of the type (II) PDE in the introduction. We consider the PDE $\Sigma=\{s+p r+t=0\}$. Then the discriminant $\Delta=1-\frac{p}{4}$. Thus, $\Sigma$ is not locally parabolic around the codimension one submanifold $\{p=4\}$.

\section{Differential Systems around nOnsubmersion Points}

In this section, we consider differential systems which satisfies the assumption 1.2. First, we give two examples of PDE. The first example satisfies the assumption 1.2, but the second example do not satisfy the assumption. From these examples, objects treated in the paper are understood.

EXAmPle 3.1. Consider the PDE $\Sigma:=\{r t-p=0\}$ which has the type (III) and (IV) points. The discriminant is $\Delta:=F_{s}{ }^{2}-\frac{1}{4} F_{r} F_{t}=-\frac{1}{4} r t$. Hence,

$$
\begin{aligned}
& \text { if } r>0, t>0 \text { or } r<0, t<0, \text { then elliptic type, } \\
& \text { if } r=0 \text { or } t=0 \text {, then parabolic type, } \\
& \text { if } r>0, t<0 \text { or } r<0, t>0 \text {, then hyperbolic type. }
\end{aligned}
$$

Then, $\Sigma$ is a smooth hypersurface of $J^{2}\left(\mathbb{R}^{2}, \mathbb{R}\right)$. But, $\left.\pi\right|_{\Sigma}$ is not submersion on the submanifold of codimension 2 defined by $r=t=0$. In practice, if we calculate matrix representations of differential maps, then the rank of matrices degenerate on $r=t=0$. To write down $D:=\left.C^{2}\right|_{\Sigma}$, we choose a local coordinate $(x, y, z, q, r, s, t)$ on $\Sigma$. Under the 
coordinate, $D=\left\{\varpi_{0}=\varpi_{1}=\varpi_{2}=0\right\}$ is given by,

$$
\begin{aligned}
& \varpi_{0}:=d z-r t d x-q d y, \\
& \varpi_{1}:=d(r t)-r d x-s d y=r d t+t d r-r d x-s d y, \\
& \varpi_{2}:=d q-s d x-t d y .
\end{aligned}
$$

On $r=t=0$, generator 1 -forms are

$$
\begin{aligned}
& \varpi_{0}:=d z-q d y, \\
& \varpi_{1}:=-s d y, \\
& \varpi_{2}:=d q-s d y .
\end{aligned}
$$

Hence, the rank of $D$ is

Rank 5 on $r=t=s=0$,

Rank 4 on $r=t=0, s \neq 0$,

Rank 4 on generic point.

Thus, $D$ is not a differential system on $r=t=s=0$, because $\varpi_{1}$ vanishes. Consequently, we do not treat $D$ around $r=t=s=0$, and consider the behavior of the system $D$ around $r=t=0, s \neq 0$.

Example 3.2. Consider the PDE $\Sigma:=\left\{r t-s^{2}=0\right\}$ which does not have the type (III) points. The discriminant is $\Delta:=F_{s}{ }^{2}-\frac{1}{4} F_{r} F_{t}=4\left(s^{2}-\frac{1}{16} r t\right)$. Hence,

$$
\begin{aligned}
& \text { if } s^{2}-\frac{1}{16} r t<0, \text { then elliptic type, } \\
& \text { if } s^{2}-\frac{1}{16} r t=0, \text { then parabolic type, } \\
& \text { if } s^{2}-\frac{1}{16} r t>0, \text { then hyperbolic type. }
\end{aligned}
$$

Then, $\Sigma$ is a smooth hypersurface of $J^{2}\left(\mathbb{R}^{2}, \mathbb{R}\right)$. But, $\left.\pi\right|_{\Sigma}$ is not submersion on the submanifold of codimension 3 defined by $r=t=s=0$. To write down $D:=\left.C^{2}\right|_{\Sigma}$, we choose a local coordinate $(x, y, z, q, r, s, t)$ on $\Sigma$. Under the coordinate, $D=\left\{\varpi_{0}=\varpi_{1}=\varpi_{2}=0\right\}$ is given by,

$$
\begin{aligned}
& \varpi_{0}:=d z-\left(r t-s^{2}\right) d x-q d y \\
& \varpi_{1}:=d\left(r t-s^{2}\right)-r d x-s d y=r d t+t d r-2 s d s-r d x-s d y, \\
& \varpi_{2}:=d q-s d x-t d y .
\end{aligned}
$$


On $r=t=s=0$, generator 1 -forms are

$$
\begin{aligned}
& \varpi_{0}:=d z-q d y, \\
& \varpi_{1}:=0, \\
& \varpi_{2}:=d q .
\end{aligned}
$$

Hence, the rank of $D$ is

Rank 5 on $r=t=s=0$

Rank 4 on generic point

Since $\Sigma$ does not have nonsubmersion points satisfying the assumption 1.2 , we do not treat this as an example.

By the definition, we have the following characterization of assumption 1.2.

Proposition 3.3. Let $\Sigma=\{F=0\} \subset J^{2}\left(\mathbb{R}^{2}, \mathbb{R}\right)$ be a second order PDE and $\pi$ : $J^{2}\left(\mathbb{R}^{2}, \mathbb{R}\right) \rightarrow J^{1}\left(\mathbb{R}^{2}, \mathbb{R}\right)$ be the natural projection. Assume that $\Sigma$ is a smooth hypersurface around $w_{0} \in \Sigma$. ( i.e. $d F \neq 0$ around $\left.w_{0}.\right)$ Then, the following conditions are equivalent.

(i) $w_{0}$ is a nonsubmersion point with respect to $\left.\pi\right|_{\Sigma}: \Sigma \rightarrow J^{1}\left(\mathbb{R}^{2}, \mathbb{R}\right)$.

(ii) $\left(F_{r}, F_{s}, F_{t}\right)_{w_{0}}=0$.

(iii) $X(F)_{w_{0}}=0$ for any $X \in k e r \pi_{*} \subset C^{2}$.

Moreover, for a nonsubmersion point $w_{0}$, the followings are equivalent

(i) $D:=\left.C^{2}\right|_{\Sigma}$ is a not differential system around a nonsubmersion point $w_{0}$.

(ii) $\frac{d F}{d x}\left(w_{0}\right)=\frac{d F}{d y}\left(w_{0}\right)=0$.

(iii) $X(F)_{w_{0}}=0$ for any $X \in C^{2}$.

(iv) $T_{w_{0}} \Sigma$ is not transverse to $C_{w_{0}}^{2}$ in $T_{w_{0}} J^{2}$. (i.e. $C_{w_{0}}^{2} \subset T_{w_{0}} \Sigma$.)

where, we used two vector fields given by

$$
\frac{d}{d x}:=\frac{\partial}{\partial x}+p \frac{\partial}{\partial z}+r \frac{\partial}{\partial p}+s \frac{\partial}{\partial q}, \quad \frac{d}{d y}:=\frac{\partial}{\partial y}+q \frac{\partial}{\partial z}+s \frac{\partial}{\partial p}+t \frac{\partial}{\partial q} .
$$

When we will prove some properties of systems, often regard the origin of $J^{2}\left(\mathbb{R}^{2}, \mathbb{R}\right)$ as nonsubmersion points, and discuss systems around the origin. We construct contact transformations on $J^{2}\left(\mathbb{R}^{2}, \mathbb{R}\right)$ sending general (including nonsubmersion) points to the origin 0 . Here, we call a diffeomorphism $\Phi: J^{2}\left(\mathbb{R}^{2}, \mathbb{R}\right) \rightarrow J^{2}\left(\mathbb{R}^{2}, \mathbb{R}\right)$ contact transformation if $\Phi$ preserves the canonical system. (i.e. $\Phi_{*} C^{2}=C^{2}$.) Let $w_{0} \in \Sigma \subset J^{2}\left(\mathbb{R}^{2}, \mathbb{R}\right)$ be a generic (nonsubmersion) point. We put $w_{0}:=\left(x_{0}, y_{0}, z_{0}, p_{0}, q_{0}, r_{0}, s_{0}, t_{0}\right)$. Then, the 
contact transformation $\Phi: J^{2}\left(\mathbb{R}^{2}, \mathbb{R}\right) \rightarrow J^{2}\left(\mathbb{R}^{2}, \mathbb{R}\right)$ sending $w_{0}$ to 0 is given by:

$$
\begin{aligned}
& X:=x-x_{0}, \\
& Y:=y-y_{0}, \\
& Z:=z-z_{0}-s_{0}\left(x-x_{0}\right)\left(y-y_{0}\right)-\frac{t_{0}}{2}\left(y-y_{0}\right)^{2}-\frac{r_{0}}{2}\left(x-x_{0}\right)^{2}-p_{0}\left(x-x_{0}\right)-q_{0}\left(y-y_{0}\right), \\
& P:=p-s_{0}\left(y-y_{0}\right)-p_{0}-r_{0}\left(x-x_{0}\right), \\
& Q:=q-s_{0}\left(x-x_{0}\right)-q_{0}-t_{0}\left(y-y_{0}\right), \\
& R:=r-r_{0}, \\
& S:=s-s_{0}, \\
& T:=t-t_{0} .
\end{aligned}
$$

It is clear that $\Phi^{*} \varpi_{i}=\varpi_{i}$ for $i=0,1,2$. The contact transformation $\Phi$ sends a reference point $w_{0}$ of the original equation $F=0$ to origin of the new equation $\bar{F}:=\left(\Phi^{-1}\right)^{*} F=0$. Therefore, we may assume a nonsubmersion point $w_{0}$ is the origin.

Example 3.4. (Continue of Example 3.1) We consider $\Sigma=\{r t-p=0\} . w_{0} \in$ $\{r=t=0, s \neq 0\} \subset \Sigma$ is a nonsubmersion pointand $D:=\left.C^{2}\right|_{\Sigma}$ is a differential system around $w_{0}$. Now, we use a contact transformation sending $w_{0}$ to 0 . We put $w_{0}:=$ $\left(x_{0}, y_{0}, z_{0}, q_{0}, 0, s_{0}, 0\right)$. According to the contact transformation (3), we consider the following transformation $\phi_{1}:\left(J^{2}\left(\mathbb{R}^{2}, \mathbb{R}\right), C^{2}\right) \rightarrow\left(J^{2}\left(\overline{\mathbb{R}^{2}}, \overline{\mathbb{R}}\right), C^{2}\right)$ :

$$
\begin{aligned}
& \bar{x}:=x-x_{0}, \\
& \bar{y}:=y-y_{0}, \\
& \bar{z}:=z-z_{0}-p_{0}\left(x-x_{0}\right)-q_{0}\left(y-y_{0}\right)-s_{0}\left(x-x_{0}\right)\left(y-y_{0}\right), \\
& \bar{p}:=p-p_{0}-s_{0}\left(y-y_{0}\right), \\
& \bar{q}:=q-q_{0}-s_{0}\left(x-x_{0}\right), \\
& \bar{r}:=r, \\
& \bar{s}:=s-s_{0}, \\
& \bar{t}:=t .
\end{aligned}
$$

Under $\phi_{1}, r t-p=0$ is transformed to

$$
\bar{y}=\frac{1}{s_{0}}\left(\bar{r} \bar{t}-\bar{p}-p_{0}\right)
$$


Moreover, we consider the following contact transformation $\phi_{2}$ preserving the origin.

$$
\begin{aligned}
& X:=\bar{y} \\
& Y:=\bar{x} \\
& Z:=\bar{z}, \\
& P:=\bar{q}, \\
& Q:=\bar{p} \\
& R:=\bar{t} \\
& S:=\bar{s}, \\
& T:=\bar{r} .
\end{aligned}
$$

By $\phi:=\phi_{2} \circ \phi_{1},\{r t-p=0\}$ is tranformed to

$$
X=\frac{1}{s_{0}}\left(T R-Q-p_{0}\right)
$$

This equation has a nonsubmersion point at 0 .

Under the preparation, we obtain some fundamental properties.

Proposition 3.5. Let $\Sigma=\{F=0\}$ be a smooth hypersurface of $J^{2}\left(\mathbb{R}^{2}, \mathbb{R}\right)$. Assume that $\Sigma$ has a nonsubmersion point $w \in \Sigma$ for $\left.\pi\right|_{\Sigma}$, and $D:=\left.C^{2}\right|_{\Sigma}$ is a differential system around $w$. Then, $D$ is regular and weakly regular. In particular, we have the following:

$$
\begin{aligned}
& D \subset \partial D \subset \partial^{2} D=T \Sigma, \\
& D \subset \partial D \subset \partial^{(2)} D=T \Sigma .
\end{aligned}
$$

where, $\operatorname{rank} \partial D=6$.

Proposition 3.6. Let $\Sigma=\{F=0\}$ be a smooth hypersurface of $J^{2}\left(\mathbb{R}^{2}, \mathbb{R}\right)$. Assume $D$ is a differential system. Then, the following conditions are equivalent.

(1) $w \in \Sigma$ is a nonsubmersion point.

(2) $\operatorname{dim} C h(D)_{w}=1$. (In regular case, $C h(D)=\{0\}$.)

(3) $\operatorname{dim} C h(\partial D)_{w}=4$. (Characteristic systems in regular case are 2-dimensional.)

Proof. First, we prove the Proposition 3.5. Since $\Sigma$ is a submanifold and $\left.\pi\right|_{\Sigma}$ is a not submersion around $w$ by the assumption, $F=0$ can be regarded as one of the following 
using the implicit function theorem:

$$
\begin{aligned}
\text { (i) } x & =f(y, z, p, q, r, s, t), \\
\text { (ii) } y & =f(x, z, p, q, r, s, t), \\
\text { (iii) } z & =f(x, y, p, q, r, s, t), \\
\text { (iv) } p & =f(x, y, z, q, r, s, t), \\
\text { (v) } q & =f(x, y, z, p, r, s, t),
\end{aligned}
$$

We may assume $w$ is the origin. It is sufficient to prove only the case (i). In practice, (i) and (ii) or (iv) and (v) are contact equivalent respectively. Moreover, if we consider the case (iv), then the system $D$ is given by

$$
\begin{aligned}
& \varpi_{0}=d z-p d x-q d y \\
& \varpi_{1}=d f-r d x-s d y \\
& \varpi_{2}=d q-s d x-t d y
\end{aligned}
$$

Since $D$ is a differential system and $w$ is a nonsubmersion point, we have $f_{x} \neq 0$ or $f_{y} \neq 0$. If $f_{x} \neq 0$, then $F=0$ can be transformed to (i) by the implicit function theorem. Also, $f_{y} \neq 0$, then $F=0$ can be transformed to (ii) by the implicit function theorem. We can apply the similar method to case (iii). Hence, we prove the statement for the case (i).

We put $\Sigma=\{x=f(y, z, p, q, r, s, t)\}$, and choose a local coordinate $(y, z, p, q, r, s, t)$ of $\Sigma$. Then, $D$ is given by:

$$
\begin{aligned}
& \varpi_{0}=d z-p d f-q d y \\
& \varpi_{1}=d p-r d f-s d y \\
& \varpi_{2}=d q-s d f-t d y
\end{aligned}
$$

where, $\left\{\varpi_{0}, \varpi_{1}, \varpi_{2}, d y, d r, d s, d t\right\}$ is a coframe of $\Sigma$. By the calculation of the structure equation of $D$,

$$
\begin{aligned}
& d \varpi_{0} \equiv 0 \quad \bmod \varpi_{0}, \varpi_{1}, \varpi_{2} \\
& d \varpi_{1}=-d r \wedge d f-d s \wedge d y \\
& d \varpi_{2}=-d s \wedge d f-d t \wedge d y
\end{aligned}
$$

Since $d \varpi_{1}$ and $d \varpi_{2}$ are linear independent, we have $\partial D=\left\{\varpi_{0}=0\right\}$. By calculation of the structure equation of $\partial D$,

$$
\begin{aligned}
d \varpi_{0} & =-d p \wedge d f-d q \wedge d y \\
& =-\left(\varpi_{1}+r d f+s d y\right) \wedge d f-\left(\varpi_{2}+s d f+t d y\right) \wedge d y \\
& =-\varpi_{1} \wedge d f-\varpi_{2} \wedge d y \\
& \neq \equiv 0 \quad \bmod \varpi_{0}, \varpi_{1} \wedge \varpi_{2}
\end{aligned}
$$


Hence, we have $\partial^{2} D=\partial^{(2)} D=T \Sigma$, and $D$ is regular and weakly regular.

Next we prove the statement of the Proposition 3.6. Here, we regard the origin 0 as a nonsubmersion point by choosing a contact transformation, and use the nonsubmersion condition $f_{r}=f_{s}=f_{t}=0$ at 0 . Then, the structure equation at 0 is given by;

$$
\begin{aligned}
& d \varpi_{0} \equiv 0 \quad \bmod \varpi_{0}, \varpi_{1}, \varpi_{2} \\
& d \varpi_{1} \equiv\left(-f_{y} d r-d s\right) \wedge d y \quad \bmod \varpi_{0}, \varpi_{1}, \varpi_{2} \\
& d \varpi_{2} \equiv\left(-f_{y} d s-d t\right) \wedge d y \quad \bmod \varpi_{0}, \varpi_{1}, \varpi_{2}
\end{aligned}
$$

From the structure equation, the Cauchy characteristic system $C h(D)$ at 0 is given by;

$$
C h(D)_{0}=\left\{\varpi_{0}=\varpi_{1}=\varpi_{2}=d y=f_{y} d r+d s=f_{y} d s+d t=0\right\} \neq\{0\}
$$

We calculate the structure equation of $\partial D$ at 0 .

$$
\begin{aligned}
d \varpi_{0} & =-d p \wedge d f-d q \wedge d y \\
& =-\varpi_{1} \wedge d f-\varpi_{2} \wedge d y \\
& \equiv-f_{y} \varpi_{1} \wedge d y-f_{q} \varpi_{1} \wedge d q-\varpi_{2} \wedge d y \\
& \equiv-\left(f_{y} \varpi_{1}+\varpi_{2}\right) \wedge d y-f_{q} \varpi_{1} \wedge \varpi_{2} \\
& \equiv-\left(f_{y} \varpi_{1}+\varpi_{2}\right) \wedge d y-f_{q} \varpi_{1} \wedge\left(\varpi_{2}+f_{y} \varpi_{1}\right) \\
& \equiv\left(f_{y} \varpi_{1}+\varpi_{2}\right) \wedge\left(-d y+f_{q} \varpi_{1}\right) \bmod \varpi_{0}
\end{aligned}
$$

Thus, we have

$$
C h(\partial D)_{0}=\left\{\varpi_{0}=f_{y} \varpi_{1}+\varpi_{2}=-d y+f_{q} \varpi_{1}=0\right\}
$$

and $\operatorname{dim} C h(\partial D)_{0}=4$. By the above discussion, we obtain (2) and (3) from (1). Conversely, if $w$ is a submersion point, it is well-known that $C h(D)=\{0\}$ and $\operatorname{dim} C h(\partial D)_{w}=2$. Consequently, we have the equivalence of three conditions, and complete the proof.

By the proof of above Proposition, we have;

Corollary 3.7. Let $\Sigma=\{F=0\}$ be a smooth hypersurface, and $D$ be a differential system. Then, $C h(D)$ is a subbundle if and only if $C h(\partial D)$ is a subbundle. Moreover, if $C h(D)($ or $C h(\partial D))$ is a subbundle, then $(\operatorname{rank} C h(D)$, rank $C h(\partial D))=(0,2)$ or $(1,4)$.

In the case of $(0,2), \Sigma=\{F=0\}$ is a regular second order PDE. Namely, $\left.\pi\right|_{\Sigma}: \Sigma \rightarrow J^{1}$ is a submersion. In the case of $(1,4)$, a local geometry of second order PDE $\Sigma$ is reduced to a submanifold theory of 5 -dimensional contact manifold $J^{1}\left(\mathbb{R}^{2}, \mathbb{R}\right)$ in the following sense.

Corollary 3.8. There exist an open set $U \subset \Sigma$, such that

$$
(U, D)=\left(\left(\left.\pi\right|_{\Sigma}\right)^{-1}\left(\Sigma^{1}\right),\left(\left.\pi\right|_{\Sigma_{*}}\right)^{-1}\left(D^{1}\right)\right)
$$

where, $\Sigma^{1}=\left.\pi\right|_{\Sigma}(\Sigma)$, and $D^{1}=\left.C^{1}\right|_{\Sigma}$. 
Proof. This result is followed by two conditions:

(i) $\left.\pi\right|_{\Sigma}$ is a locally nonsubmersion.

(ii) $\left.C h(\partial D) \supset k e r \pi\right|_{\Sigma_{*}}=\left\{\frac{\partial}{\partial r}, \frac{\partial}{\partial s}, \frac{\partial}{\partial t}\right\}$.

In the case of $(0,2)$, it is well-known that the leaf space by Cauchy characteristic system $C h(\partial D)$ is $\Sigma / C h(\partial D) \cong J^{1}\left(\mathbb{R}^{2}, \mathbb{R}\right)$. In the case of $(1,4)$, the leaf space $\Sigma / C h(D)$ is locally isomorphic to $\left.\pi\right|_{\Sigma} ^{-1}\left(\Sigma^{1} / C h\left(D^{1}\right)\right)$. Also, the leaf space $\Sigma / C h(\partial D)$ is locally isomorphic to the 3-dimensional contact manifold. From these facts, under the assumption of Proposition 3.6, nonsubmersion points as a contact geometry of second order PDE corresponds to singular points of $C h(D)$ and $C h(\partial D)$ as a subbundle.

The rest of this section, we study the symbol algebra introduced by N. Tanaka at a nonsubmersion point.([Tan 2]) For the purpose, we define the symbol algebra. Let $D$ be a weakly regular differential system on $\Sigma$ such that

$$
T \Sigma \supset D^{-\mu} \supset D^{-(\mu-1)} \supset \cdots \supset D^{-1}=: D
$$

For all $x \in \Sigma$, we put $\mathfrak{g}_{-1}(x):=D^{-1}(x)=D(x), \mathfrak{g}_{p}(x):=D^{p}(x) / D^{p+1}(x)$, and

$$
\mathfrak{m}(x):=\bigoplus_{p=-1}^{-\mu} \mathfrak{g}_{p}(x) .
$$

Then, $\operatorname{dim} \mathfrak{m}(x)=\operatorname{dim} \Sigma$. For $X \in \mathfrak{g}_{p}(x), Y \in \mathfrak{g}_{q}(x)$, Lie bracket $[X, Y] \in \mathfrak{g}_{p+q}(x)$ is defined by;

Let $\tilde{X} \in \mathcal{D}^{p}, \tilde{Y} \in \mathcal{D}^{q}$ be extensions $\left(\tilde{X}_{x}=X, \tilde{Y}_{x}=Y\right)$, then $[\tilde{X}, \tilde{Y}] \in \mathcal{D}^{p+q}$ and $[X, Y]:=[\tilde{X}, \tilde{Y}]_{x} \in \mathfrak{g}_{p+q}(x)$ does not depend on the extensions, because of the equation

$$
[f \tilde{X}, g \tilde{Y}]=f g[\tilde{X}, \tilde{Y}]+f(\tilde{X} g) \tilde{Y}-g(\tilde{Y} f) \tilde{X} \quad\left(f, g \in C^{\infty}(\Sigma)\right) .
$$

An algebra $\mathfrak{m}(x)$ is a nilpotent graded Lie algebra.

Definition 3.9. $(\mathfrak{m}(x),[]$,$) is called symbol algebra of (\Sigma, D)$ at $x$.

For differential systems satisfying the assumption 1.2, we have the following result with respect to a symbol algebra at a nonsubmersion point.

TheOREM 3.10. Let $\Sigma=\{F=0\}$ be a smooth hypersurface of $J^{2}\left(\mathbb{R}^{2}, \mathbb{R}\right)$. Assume that $\Sigma$ has a nonsubmersion point $w \in \Sigma$ for $\left.\pi\right|_{\Sigma}$, and $D:=\left.C^{2}\right|_{\Sigma}$ is a differential system around w. Then, the symbol algebra $\mathfrak{m}(w)$ at $w$ is locally isomorphic to $\mathfrak{m}, \mathfrak{m}=\mathfrak{g}_{-3} \oplus \mathfrak{g}_{-2} \oplus \mathfrak{g}_{-1}$ and bracket relations are given by;

$\left[X_{r}, X_{x}\right]=X_{1}, \quad\left[X_{s}, X_{x}\right]=X_{2}, \quad\left[X_{1}, X_{x}\right]=X_{0}$ 
the other is trivial,

where, $\left\{X_{0}, X_{1}, X_{2}, X_{x}, X_{r}, X_{s}, X_{t}\right\}$ are basis, and

$$
\begin{aligned}
& \mathfrak{g}_{-1}=\left\{X_{x}, X_{r}, X_{s}, X_{t}\right\}, \\
& \mathfrak{g}_{-2}=\left\{X_{1}, X_{2}\right\}, \\
& \mathfrak{g}_{-3}=\left\{X_{0}\right\} .
\end{aligned}
$$

Proof. We regard the origin 0 as a nonsubmersion point $w$. Also, it is sufficient to prove the statement for $\Sigma=\{x=f(y, z, p, q, r, s, t)\}$ similar to Proposition 3.6 using the implicit function theorem. From weak regularity of $D$ (i.e. Proposition 3.5.), a symbol algebra at 0 has depth 3 , that is

$$
\mathfrak{m}(0)=\mathfrak{g}_{-3} \oplus \mathfrak{g}_{-2} \oplus \mathfrak{g}_{-1} .
$$

So, we determine bracket relations of two cases:

$$
[1]\left[\mathfrak{g}_{-1}, \mathfrak{g}_{-1}\right]=\mathfrak{g}_{-2}, \quad[2]\left[\mathfrak{g}_{-1}, \mathfrak{g}_{-2}\right]=\mathfrak{g}_{-3}
$$

In the case [1], we use the structure equation;

$$
\begin{aligned}
& d \varpi_{0} \equiv 0 \\
& d \varpi_{1} \equiv-\left(f_{y} d r+d s\right) \wedge d y \\
& d \varpi_{2} \equiv-\left(f_{y} d s+d t\right) \wedge d y \quad \bmod \varpi_{0}, \varpi_{1}, \varpi_{2}
\end{aligned}
$$

On the other hand, we use the structure equation for the case [2],

$$
d \varpi_{0} \equiv-\left(f_{y} \varpi_{1}+\varpi_{2}\right) \wedge d y \quad \bmod \varpi_{0}, \varpi_{1} \wedge \varpi_{2}
$$

Now, we choose a new coframe;

$$
\left\{\varpi_{0}, \varpi_{1}, \hat{\varpi}_{2}, d y, d r, \varpi_{s}, \varpi_{t}\right\}
$$

where, $\hat{\varpi}_{2}:=\varpi_{2}+f_{y} \varpi_{1}, \varpi_{s}:=f_{y} d r+d s$, and $\varpi_{t}:=f_{y}\left(f_{y} d r+d s\right)+\left(f_{y} d s+d t\right)$. Under the coframe, the above structure equations are written as

$$
\begin{aligned}
& d \varpi_{0} \equiv 0 \\
& d \varpi_{1} \equiv-\varpi_{s} \wedge d y \\
& d \hat{\varpi}_{2} \equiv-\varpi_{t} \wedge d y \quad \bmod \varpi_{0}, \varpi_{1}, \hat{\varpi}_{2} \\
& d \varpi_{0} \equiv-\hat{\varpi}_{2} \wedge d y \quad \bmod \varpi_{0}, \varpi_{1} \wedge \hat{\varpi}_{2}
\end{aligned}
$$

Let $\left\{X_{0}, X_{1}, X_{2}, X_{y}, X_{r}, X_{s}, X_{t}\right\}$ be the dual frame of the coframe $\left\{\varpi_{0}, \varpi_{1}, \hat{\varpi}_{2}, d y, d r, \varpi_{s}, \varpi_{t}\right\}$. Then, bracket products of a symbol algebra at 0 is given by;

$$
\left[X_{s}, X_{y}\right]=X_{1}, \quad\left[X_{t}, X_{y}\right]=X_{2}, \quad\left[X_{2}, X_{y}\right]=X_{0}
$$

other is trivial 
In practice, we put

$$
\left[X_{s}, X_{y}\right]=A_{1} X_{1}+A_{2} X_{2} \in \mathfrak{g}_{-2} \quad\left(A_{1}, A_{2} \in \mathbb{R}\right) .
$$

Then

$$
\begin{aligned}
d \varpi_{1}\left(X_{s}, X_{y}\right) & =X_{s}\left(\varpi_{1}\left(X_{y}\right)\right)-X_{y}\left(\varpi_{1}\left(X_{s}\right)\right)-\varpi_{1}\left(\left[X_{s}, X_{y}\right]\right) \\
& =-\varpi_{1}\left(\left[X_{s}, X_{y}\right]\right) \\
& =-A_{1} .
\end{aligned}
$$

On the other hand

$$
\begin{aligned}
d \varpi_{1}\left(X_{s}, X_{y}\right) & =-\varpi_{s}\left(X_{s}\right) d y\left(X_{y}\right)+d y\left(X_{s}\right) \varpi_{s}\left(X_{y}\right) \\
& =-1 .
\end{aligned}
$$

Therefore, $A_{1}=1$. From the same argument, we get $A_{2}=0$. Moreover, we have all bracket relations using similar method.

The obtained symbol algebra equals to the well known symbol of first order regular PDE. Let $G=0$ be a first order regular PDE. That is, $R=\{G(x, y, z, p, q)=0\}$ is a smooth hypersurface of $J^{1}\left(\mathbb{R}^{2}, \mathbb{R}\right)$, and $T R$ is transverse to the canonical system $C^{1}$ on $J^{1}\left(\mathbb{R}^{2}, \mathbb{R}\right)$. Then we consider $G=0$ as a second order PDE. Namely $R:=\{G=0\} \subset$ $J^{2}\left(\mathbb{R}^{2}, \mathbb{R}\right)$. Then we have

Corollary 3.11. The symbol algebra $\mathfrak{m}(w)$ of $D:=\left.C^{2}\right|_{R}$ at $w \in R$ is isomorphic to $\mathfrak{m}$ of Theorem 3.10 .

REMARK 3.12. From these results, a geometry of system corresponding to second order PDE at a nonsumbersion point is obtained from first order regular PDE. However, the fact holds only at a nonsubmersion point, because our results is obtained by pointwise discussions. In practice, a differential system satisfying the assumption 1.2 is weakly regular, but it is not always strongly regular in the sense of Tanaka. That is, symbol algebras are not equivalent in neighborhood of a nonsubmersion point. It is obvious that the symbol algebras at regular points are different from the above $\mathfrak{m}$. Summarize, a geometry in the present note is not contact geometry of first order as local geometry, and it is a (local) contact geometry of second order around a nonsubmerion point corresponding to second order PDE.

\section{A NEW INVARIANT OF SECOND ORDER PDE}

In the previous section, we studied fundamental properties of differential systems around a nonsubmersion point $w$, and showed that a symbol algebra at $w$ is determined uniquely. Hence, we do not discriminate systems satisfying the assumption 1.2 under symbol algebras. We introduce a new invariant of second order PDE in this section, and give examples which are not equivalent using this invariant. 
REMARK 4.1. In the definition of the our invariant, we do not need to assume the assumption 1.2.

Let $\Sigma:=\{F=0\}$ be a smooth hypersurface of $J^{2}\left(\mathbb{R}^{2}, \mathbb{R}\right)$. We fix a base point $w \in \Sigma$. For an any open neighborhood $U$ of $w, U$ is decomposed as follows.

$$
U=U_{h} \cup U_{e} \cup U_{p} \cup U_{\text {sing }} \quad \text { (disjoint union), }
$$

where, components are given by

$$
\begin{aligned}
U_{h} & :=\left\{v \in U \mid F_{s}^{2}-\frac{1}{4} F_{r} F_{t}(v)>0\right\} \quad: \text { hyperbolic type } \\
U_{e} & :=\left\{v \in U \mid F_{s}^{2}-\frac{1}{4} F_{r} F_{t}(v)<0\right\} \quad: \text { elliptic type } \\
U_{p} & :=\left\{v \in U \mid F_{s}^{2}-\frac{1}{4} F_{r} F_{t}(v)=0,\left(F_{r}, F_{s}, F_{t}\right)_{v} \neq 0\right\} \quad: \text { parabolic type } \\
U_{\text {sing }} & :=U \backslash\left(U_{h} \cup U_{e} \cup U_{p}\right)
\end{aligned}
$$

For an each component, we define an equivalence relation. We put $K_{U}:=U_{h}$ or $U_{e}$ or $U_{p}$ or $U_{\text {sing }}$. Then, $w_{1} \sim w_{2}\left(w_{1}, w_{2} \in K_{U}\right)$ is defined as follows.

There exist a continuous curve $c:[0,1] \rightarrow K_{U}$ s.t. $c(0)=w_{1}, c(1)=w_{2}$.

Let $\#\left(K_{U} / \sim\right)$ be the number of elements of the quotient space $K_{U} / \sim$.

Now, we fix a diffeomorphism $J^{2}\left(\mathbb{R}^{2}, \mathbb{R}\right) \cong \mathbb{R}^{8}$. Then, the standard metric on $\mathbb{R}^{8}$ induce a metric on $J^{2}\left(\mathbb{R}^{2}, \mathbb{R}\right)$ using the diffeomorphism. (The induced norm is not standard for the jet space.) We choose the following neighborhood as $U$.

$$
U:=B_{r}(w)=\{v \in \Sigma|| v-w \mid<r\},
$$

where, $|\cdot|$ is the restriction of the norm on $J^{2}\left(\mathbb{R}^{2}, \mathbb{R}\right)$ into $\Sigma$. We decompose $U$ similar to $(4)$ :

$$
U=B_{r}^{H}(w) \cup B_{r}^{E}(w) \cup B_{r}^{P}(w) \cup B_{r}^{S i n g}(w) \quad \text { (disjoint union), }
$$

where,

$$
\begin{aligned}
B_{r}^{H}(w) & :=\left\{v \in B_{r}(w) \mid F_{s}{ }^{2}-\frac{1}{4} F_{r} F_{t}(v)>0\right\} \quad: \text { hyperbolic type } \\
B_{r}^{E}(w) & :=\left\{v \in B_{r}(w) \mid F_{s}{ }^{2}-\frac{1}{4} F_{r} F_{t}(v)<0\right\} \quad: \text { elliptic type } \\
B_{r}^{P}(w) & :=\left\{v \in B_{r}(w) \mid F_{s}{ }^{2}-\frac{1}{4} F_{r} F_{t}(v)=0,\left(F_{r}, F_{s}, F_{t}\right) \neq 0\right\} \quad: \text { parabolic type } \\
B_{r}^{\text {Sing }}(w) & :=B_{r}(w) \backslash\left(B_{r}^{H}(w) \cup B_{r}^{E}(w) \cup B_{r}^{P}(w)\right) .
\end{aligned}
$$

Moreover, we define an equivalence relation for an each component in (6) similar to (5), and consider quotient spaces of these components by the relation. Then, we consider 
numbers:

$$
\begin{aligned}
& H_{|\cdot|}(w):=\lim _{r \rightarrow 0} \#\left(B_{r}^{H} / \sim\right), \quad E_{|\cdot|}(w):=\lim _{r \rightarrow 0} \#\left(B_{r}^{E} / \sim\right), \\
& P_{|\cdot|}(w):=\lim _{r \rightarrow 0} \#\left(B_{r}^{P} / \sim\right), \quad S_{|\cdot|}(w):=\lim _{r \rightarrow 0} \#\left(B_{r}^{\text {Sing }} / \sim\right),
\end{aligned}
$$

where, if a limit is not determined, we put a value $\infty$. $H, E, P, S$ values at $\{0,1,2, . ., \infty\}$. These numbers does not depend on the contact isomorphisms of $(\Sigma, D)$, but depend on the metric on $J^{2}\left(\mathbb{R}^{2}, \mathbb{R}\right)$ under the identification $J^{2}\left(\mathbb{R}^{2}, \mathbb{R}\right) \cong \mathbb{R}^{8}$. Hence, we define the following invariant of $(\Sigma, D)$ at $w$.

DEFINITION 4.2. We put

$$
\begin{aligned}
H(w) & :=\min _{J^{2} \cong \mathbb{R}^{8}}\left(\lim _{r \rightarrow 0} \#\left(B_{r}^{H} / \sim\right)\right), \\
E(w) & :=\min _{J^{2} \cong \mathbb{R}^{8}}\left(\lim _{r \rightarrow 0} \#\left(B_{r}^{E} / \sim\right)\right), \\
P(w) & :=\min _{J^{2} \cong \mathbb{R}^{8}}\left(\lim _{r \rightarrow 0} \#\left(B_{r}^{P} / \sim\right)\right), \\
S(w) & :=\min _{J^{2} \cong \mathbb{R}^{8}}\left(\lim _{r \rightarrow 0} \#\left(B_{r}^{\text {Sing }} / \sim\right)\right) .
\end{aligned}
$$

The quadruple $(H, E, P, S)_{w}$ denotes the vector constructed by $H(w), E(w), P(w)$, and $S(w)$.

Remark 4.3. The value $(H, E, P, S)_{w}$ does not depend on the identification $J^{2}\left(\mathbb{R}^{2}, \mathbb{R}\right) \cong$ $\mathbb{R}^{8}$. Thus, it is an invariant of $(\Sigma, D)$ at $w$.

By the definition, the following fundamental theorem which characterize flat model.

ThEOREM 4.4. Let $\Sigma=\{F=0\} \subset J^{2}$ be a second order PDE. Then,

(1) $(1,0,0,0)_{w} \Longleftrightarrow \Sigma$ is locally hyperbolic around $w$.

(2) $(0,1,0,0)_{w} \Longleftrightarrow \Sigma$ is locally elliptic around $w$.

(3) $(0,0,1,0)_{w} \Longleftrightarrow \Sigma$ is locally parabolic around $w$.

where, $w$ is a point in $\Sigma$.

From this theorem, we can say that our invariant for systems is a tool to study systems the case (II), (III) as in the introduction. We give two distinct examples using $(H, E, P, S)_{w}$.

EXAMPLE 4.5. (Continue of Example 3.1)

We consider $\Sigma=\{r t-p=0\}$. A point $w \in\{r=t=0, s \neq 0\} \subset \Sigma$ is a nonsubmersion point and $D:=\left.C^{2}\right|_{\Sigma}$ is a differential system around $w$. Fix a base point $w$ on $\{r=t=0, s \neq 0\}$. Recall that the discriminant is $\Delta=-\frac{1}{4} r t$. Hence, points on $\{r=0, t \neq 0\} \cup\{r \neq 0, t=0\}$ are parabolic type. Also, two hyperbolic and elliptic parts 
appear in connected components divided by $\{r=0\}$ and $\{t=0\}$. (cf. Example 3.1) Thus, we have $(H, E, P, S)_{w}=(2,2,4,1)$ for this case. (Precisely, see the Figure 1.)

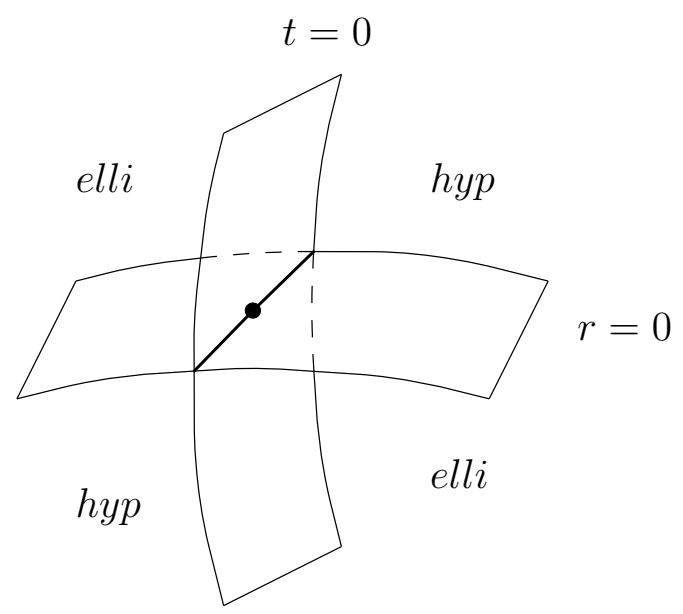

Figure 1

EXAMPLE 4.6. We consider $\Sigma=\{p s-x=0\}$. We have nonsubmersion points on $\{p=0\} \subset \Sigma$. Now, we take a nonsubmersion point $w=(0, \ldots, 0)$ which give a differential system $D$ around 0 . The discriminant of the equation is $\Delta=p^{2}$. By these condition, there is not a parabolic point. Because, if $\Delta=0$, then $p=0$ and points on $\{p=0\}$ are nonsubmerion points. Also, there is not an elliptic point by $\Delta>0$. Hyperbolic points appear in connected component divided by $\{p=0\}$. Summarize, we have $(H, E, P, S)_{w}=$ $(2,0,0,1)$.

For second order PDEs of the following special form, we characterize differential systems $(\Sigma, D)$ using the our invariant.

TheOREM 4.7. Let $\Sigma=\{F=0\}$ be a smooth hypersurface given by;

$$
F:=f-\left(a_{1} x+a_{2} y+a_{3} z+a_{4} p+a_{5} q+a_{6}\right)
$$

where, $f$ is a monomial of degree two with respect to $r, s, t$, and $\left(a_{1}, . ., a_{5}\right) \neq 0$. Then, $(H, E, P, S)_{w}$ at a nonsubmersion point $w$ satisfying the assumption 1.2 depends only on $F$. (That is, $(H, E, P, S)_{w}$ does not depend on the choice of nonsubmersion points.) Moreover, values of $(H, E, P, S)_{w}$ for $\Sigma$ are one of the followings.

(1) $(2,0,2,1)_{w}$ for $f=r s$ or $t s$,

(2) $(2,2,4,1)_{w}$ for $f=r t$,

(3) $(0,0,2,1)_{w}$ for $f=r^{2}$ or $t^{2}$,

(4) $(2,0,0,1)_{w}$ for $f=s^{2}$.

Proof. First, we prove (1). Since $f=r s$ and $f=t s$ are contact equivalent, we prove (1) for $f=r s$. From the condition $\left(a_{1}, . ., a_{5}\right) \neq 0, \Sigma$ is a smooth hypersurface. 
(This fact is similar in other cases.) By $\left(F_{r}, F_{s}, F_{t}\right)=(s, r, 0)$, points on $\{r=s=0\}$ are nonsubmersion points. When we take an any point $v, D:=\left.C^{2}\right|_{\Sigma}$ is a differential system around $v$ if and only if $\frac{d F}{d x} \neq 0$ or $\frac{d F}{d y} \neq 0$ from the proposition 3.3. We fix a base point $w$ satisfying the condition. Since $\Delta=r^{2}$, there is not an elliptic point. Also, points on $\{r=0, s>0\} \cup\{r=0, s<0\}$ are parabolic. Moreover, hyperbolic points appear in $\{r \neq 0\}$ which has two connected components. Thus, we have $(H, E, P, S)_{w}=(2,0,2,1)$. For the case (2), we omit the proof of the case (2), because of the same discussion to the example $\{r t-p=0\}$. Next, we prove (3) for $f=r^{2} .\left(f=r^{2}\right.$ and $f=t^{2}$ are contact equivalent.) By the definition $F=r^{2}-\left(a_{1} x+a_{2} y+a_{3} z+a_{4} p+a_{5} q+a_{6}\right)$, we have nonsubmersion points on $\{r=0\} \subset \Sigma$. Also, $\Delta=0$ and we do not have hyperbolic and elliptic points. Moreover, we have parabolic points in $\{r \neq 0\}$ which has two connected components. Consequently, we obtain $(H, E, P, S)_{w}=(0,0,2,1)$ at a nonsubmersion point $w$. Finally, we prove (4). From the expression $F=s^{2}-\left(a_{1} x+a_{2} y+a_{3} z+a_{4} p+a_{5} q+\right.$ $\left.a_{6}\right)$, we have nonsubmersion points on $\{s=0\} \subset \Sigma$. Hence, we do not have parabolic points. Because, if $\Delta=0$, then $s=0$ and this condition give nonsubmersion points. Also, $\Delta=4 s^{2}$ and we do not have elliptic points. Moreover, we have hyperbolic points on $\{s=0\}$ which has two connected components. Thus, we have $(H, E, P, S)_{w}=(2,0,0,1)_{w}$ at a nonsubmersion point $w$.

From the above proof, it is clear that $(H, E, P, S)_{w}$ does not depend on choice of nonsubmersion points. Hence, we obtain the statement.

\section{REFERENCES}

[BCG3] R. Bryant, S. S. Chern, R. Gardner, H. Goldscmidt, P. Griffiths, Exterior Differential Systems, MSRI Publ. vol. 18, Springer Verlag, Berlin (1991).

[Car] E. Cartan, Les systèmes de Pfaff à cing variables et les équations aux dérivées partielles du second ordre, Ann. École Normale, 27 (1910), 109-192.

[CKW] J. Clelland, M. Kossowski,G.R. Wilkens, Second-order type-changing evolution equations with first-order intermediate equations, J. Differential Equations, 244, (2008) no.2, 242-273.

[HIIY] K. Hayakawa, G. Ishikawa, S. Izumiya, K. Yamaguchi, Classification of generic integral diagrams and first order ordinary differential equations, Internat. J. Math. Vol. 5, No. 4, (1994) 447-489.

[NS] T. Noda, K. Shibuya, Second order type-changing PDE for a scalar function on a plane, in preparation.

[S] K. Shibuya, On the prolongation of 2-jet space of 2 independent and 1 dependent variables, to appear in Hokkaido Math. J.

[Tak] M. Takahashi, Bifurcations of ordinary differential equations of clairaut type, J. Differential Equations, 190, (2003) no.2, 579-599.

[Tan1] N. Tanaka On generalized graded Lie algebras and geometric structures I, J. Math. Soc. Japan, 19 (1967), 215-254.

[Tan2] N. Tanaka, On the equivalence problems associated with simple graded Lie algebras, Hokkaido Math. J. 8 (1979), no. 1, 23-84.

[Y1] K. Yamaguchi, Contact geometry of higher order, Japan. J. Math., 8 (1982), 109-176.

[Y2] K. Yamaguchi, Geometrization of jet bundles, Hokkaido Math. J. 12 (1983), 27-40. 
[Y3] K. Yamaguchi, Differential systems associated with simple graded Lie algebras, Advanced Studies in Pure Math., 22 (1993), 413-449.

[Y4] K. Yamaguchi, Contact geometry of second order, Hokkaido university preprint series in mathematics No. 927

\section{TAKAHIRO NODA}

Graduate School of Mathematics

NAGOYA UNIVERSity

Chikusa-Ku, NagoYa 464-8602

JAPAN

Kazuhiro Shibuya

Department of Mathematics

Graduate School of Science

HOKKAIDO UNIVERSITY

SAPPORO 060-0810

JAPAN

E-mail address: m04031x@math.nagoya-u.ac.jp

E-mail address: shibuya@math.sci.hokudai.ac.jp 